\title{
Steps towards a high precision solar rotation profile: Results from SDO/AIA coronal bright point data
}

\author{
D. Sudar ${ }^{1}$, I. Skokić ${ }^{2}$, R. Brajša ${ }^{1}$, and S. H. Saar ${ }^{3}$ \\ ${ }^{1}$ Hvar Observatory, Faculty of Geodesy, University of Zagreb, Kačićeva 26, 10000 Zagreb, Croatia \\ e-mail: davor.sudar@gmail.com \\ 2 Cybrotech Ltd, Bohinjska 11, 10000 Zagreb, Croatia \\ ${ }^{3}$ Harvard-Smithsonian Center for Astrophysics, 60 Garden Street, Cambridge, MA 02138, USA \\ Received 5 September 2014 / Accepted 23 December 2014
}

\section{ABSTRACT}

\begin{abstract}
Context. Coronal bright points (CBP) are ubiquitous small brightenings in the solar corona associated with small magnetic bipoles. Aims. We derive the solar differential rotation profile by tracing the motions of CBPs detected by the Atmospheric Imaging Assembly (AIA) instrument aboard the Solar Dynamics Observatory (SDO). We also investigate problems related to the detection of CBPs resulting from instrument and detection algorithm limitations.

Methods. To determine the positions and identification of CBPs we used a segmentation algorithm. A linear fit of their central meridian distance and latitude vs time was used to derive velocities.

Results. We obtained 906 velocity measurements in a time interval of only 2 days. The differential rotation profile can be expressed as $\omega_{\text {rot }}=\left(14^{\circ} .47 \pm 0.10+\left(0^{\circ} 6 \pm 1^{\circ} .0\right) \sin ^{2}(b)+\left(-4^{\circ} .7 \pm 1.7\right) \sin ^{4}(b)\right) \mathrm{d}^{-1}$. Our result is in agreement with other work and it comes with reasonable errors in spite of the very short time interval used. This was made possible by the higher sensitivity and resolution of the AIA instrument compared to similar equipment as well as high cadence. The segmentation algorithm also played a crucial role by detecting so many CBPs, which reduced the errors to a reasonable level.

Conclusions. Data and methods presented in this paper show a great potential for obtaining very accurate velocity profiles, both for rotation and meridional motion and, consequently, Reynolds stresses. The amount of CBP data that could be obtained from this instrument should also provide a great opportunity to study changes of velocity patterns with a temporal resolution of only a few months. Other possibilities are studies of evolution of CBPs and proper motions of magnetic elements on the Sun.
\end{abstract}

Key words. Sun: rotation - Sun: corona - Sun: activity

\section{Introduction}

We present a new solar rotation profile obtained by tracing the motions of coronal bright points (CBPs) observed by the Atmospheric Imaging Assembly (AIA) instrument on board the Solar Dynamics Observatory (SDO) satellite (Lemen et al. 2012).

The most frequently used and oldest tracers of the solar differential rotation profile are sunspots (Newton \& Nunn 1951; Howard et al. 1984; Balthasar et al. 1986b; Brajša et al. 2002a). One of the advantages of using sunspots is very long time coverage. On the other hand, there are numerous disadvantages: sunspots have complex and evolving structure, their distribution in latitude is highly non-uniform, and they do not extend to higher solar latitudes. The number of sunspots is also highly variable during the solar cycle which makes measurements of solar differential rotation profiles almost impossible during solar minimum.

Coronal bright points are more uniformly distributed in latitude and are numerous in all phases of the solar cycle. They also extend over all solar latitudes. They have been used as tracers of solar rotation since the beginning of the space age (Dupree \& Henze 1972). In recent years there have been numerous studies investigating solar differential rotation by using CBPs as tracers. Kariyappa (2008), Hara (2009) used Yohkoh/SXT data, while Brajša et al. (2001, 2002b, 2004), Vršnak et al. (2003), Wöhl et al. (2010) used SOHO/EIT observations in $28.4 \mathrm{~nm}$ channel, and Karachik et al. (2006) used 19.4 nm SOHO/EIT channel.
Kariyappa (2008) also used Hinode/XRT full-disk images to determine the solar rotation profile.

Other tracers are used as well, for example magnetic fields (Wilcox \& Howard 1970; Snodgrass 1983; Komm et al. 1993) and $\mathrm{H} \alpha$ filaments (Brajša et al. 1991). Apart from tracers, Doppler measurements can also be used (Howard \& Harvey 1970; Ulrich et al. 1988; Snodgrass \& Ulrich 1990).

Helioseismic measurements also show differential rotation below the photosphere all the way down to the bottom of the convective zone (Kosovichev et al. 1997; Schou et al. 1998). Further down, the rotation profile becomes uniform for all latitudes (see e.g. Howe 2009).

For further details about solar rotation, its importance for solar dynamo models, and a comparison of rotation measurements between different sources, see the reviews by Schröter (1985), Howard (1984), Beck (2000), Ossendrijver (2003), Rüdiger \& Hollerbach (2004), Stix (2004), Howe (2009) and Rozelot \& Neiner (2009).

In this work we use CBP data obtained by SDO/AIA over only two days to assess the quality of the data, identify sources of errors and calculate the solar differential rotation profile. We will also investigate the possibility of using CBP data from SDO/AIA for further studies of other related phenomena (meridional flow, rotation velocity residuals, and Reynolds stress).

Coronal bright point data from SDO/AIA have also been in other works. Lorenc et al. (2012) discussed rotation of the solar corona based on 69 structures from 674 images detected in the $9.4 \mathrm{~nm}$ channel using an interactive method of detection. 
Dorotovič et al. (2014) presented a hybrid algorithm for detection and tracking of CBPs. McIntosh et al. (2014b) used detection algorithm presented in their previous paper (McIntosh \& Gurman 2005) to identify CBPs in the SDO/AIA $19.4 \mathrm{~nm}$ channel and correlate their properties with those of giant convective cells. Using more SDO/AIA data and extending analysis back to SOHO era, McIntosh et al. (2014a) concluded that CBPs almost exclusively form around the vertices of giant convective cells.

\section{Data and reduction methods}

We used data from the AIA instrument on board the SDO satellite (Lemen et al. 2012). The spatial resolution of the instrument is $\approx 0.6^{\prime \prime} /$ pixel. For comparison, SOHO/EIT resolution is $2.629^{\prime \prime} /$ pixel, while Hinode/XRT has a resolution of $1.032^{\prime \prime} /$ pixel.

To obtain positional information for the CBPs, we employed a segmentation algorithm which uses the $19.3 \mathrm{~nm}$ AIA channel data to search for localized, small intensity enhancements in the EUV compared to a smoothed background intensity. More details about the detection algorithm, which is similar to the algorithm by McIntosh \& Gurman (2005), can be found in Martens et al. (2012).

This resulted in measurements of 66842 positions of 13646 individual CBPs covering two days (1 and 2 January 2011). The time interval between two successive images was $10 \mathrm{~min}$.

In the top panel of Fig. 1 we show the distribution of detected CBPs and compare it to the full disk image of the Sun in the $19.3 \mathrm{~nm}$ channel obtained on 1 January 2011 (bottom panel of the same figure). In the bottom panel, white circles show CBPs that were detected on one image by the segmentation algorithm. We can see that there are not many CBPs in active regions, partly because of difficulties in detecting them against such bright and variable backgrounds.

The segmentation algorithm provides coordinates in pixels (centroids of CBPs on the image) and we converted them to heliographic coordinates taking into account the current solar distance given in FITS files (Roša et al. 1995, 1998). Positions of objects near the solar limb are fairly inaccurate. Limiting the data to $\pm 58^{\circ}$ from the centre of the Sun or $\approx 0.85 R_{\odot}$ of the projected solar disk removes this problem (cf. Stark \& Wöhl 1981; Balthasar et al. 1986a).

As can be seen from Fig. 2, the calculated velocities show some scatter. This scatter arises because the shifts are fairly small at our 10 min cadence, and there can be significant variations in brightness and structure of CBPs which influences the calculation of the centroid points. Nevertheless, trends are visible, especially in azimuthal motion which is known to be a significantly larger effect. The dominant azimuthal motion led us to approximate the CBP motion with a linear fit to calculate the velocities,

$$
\begin{aligned}
& \omega_{\mathrm{syn}}= \frac{N \sum_{i=1}^{N} l_{i} t_{i}-\sum_{i=1}^{N} l_{i} \sum_{i=1}^{N} t_{i}}{N \sum_{i=1}^{N} t_{i}^{2}-\left(\sum_{i=1}^{N} t_{i}\right)^{2}}, \\
& \omega_{\mathrm{mer}}=\frac{N \sum_{i=1}^{N} b_{i} t_{i}-\sum_{i=1}^{N} b_{i} \sum_{i=1}^{N} t_{i}}{N \sum_{i=1}^{N} t_{i}^{2}-\left(\sum_{i=1}^{N} t_{i}\right)^{2}}
\end{aligned}
$$

where $\omega_{\text {syn }}$ is the synodic rotational velocity, $\omega_{\text {mer }}$ is the meridional angular velocity, $l_{i}$ is the central meridian distance (CMD)
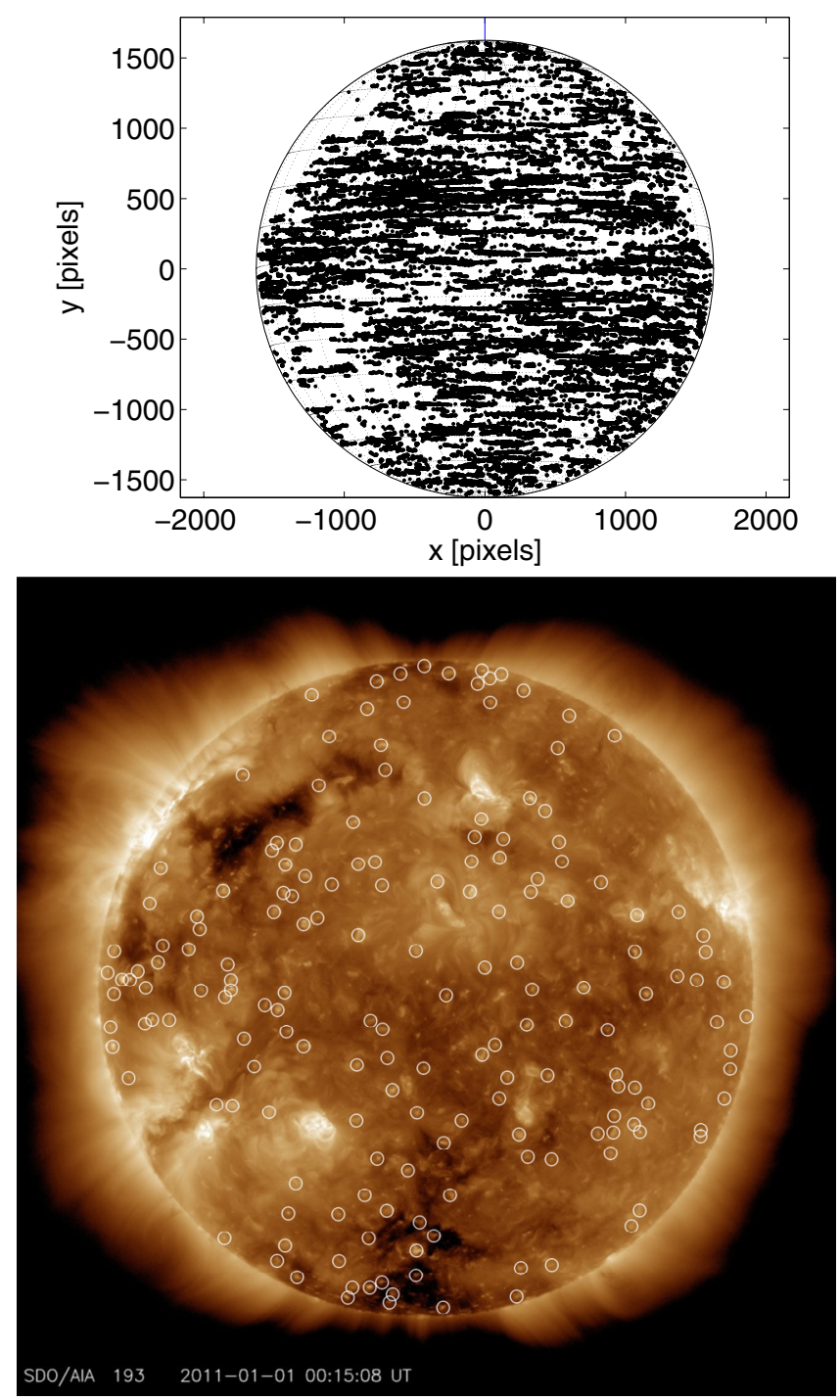

Fig. 1. Distribution of CBPs detected by the segmentation algorithm (top panel) and image of the Sun in the $19.3 \mathrm{~nm}$ channel obtained by SDO/AIA on 1st of January 2011. White circles show detected CBPs on this image (bottom panel).

and $b_{i}$ is the latitude of each measurement for a single CBP. We have also removed all CBPs which had fewer than 10 measurements of position in order for linear fits to be more robust. This is equivalent to $100 \mathrm{~min}$ or about $1^{\circ}$ at the equator. To obtain the true rotation of CBPs on the Sun, we convert synodic velocities to sidereal using Eq. (7) from Skokić et al. (2014).

Trying to identify the same object on subsequent images with an automatic method is bound to result in some misidentifications. The resulting velocities are usually very large and can easily be removed by applying a simple velocity filter. Even the human factor can introduce such errors. For example, Sudar et al. (2014) analysed solar rotation residuals and meridional motions of sunspot groups from the Greenwich Photoheliographic Results and found that they had to use a filter $8<\omega_{\text {rot }}<19^{\circ} \mathrm{d}^{-1}$ for rotational velocity in order to eliminate these erroneous measurements. The Greenwich Photoheliographic Results catalogue is being investigated and revised partly in order to remove such problems (Willis et al. 2013a,b; Erwin et al. 2013). In this work we also used a $8<\omega_{\text {rot }}<19^{\circ} \mathrm{d}^{-1}$ filter for rotational velocities to remove such outliers. In addition, we applied a meridional velocity filter of $-4<\omega_{\text {mer }}<4^{\circ} \mathrm{d}^{-1}$ to remove further outliers. 
D. Sudar et al.: Steps towards a high precision solar rotation profile: Results from SDO/AIA coronal bright point data
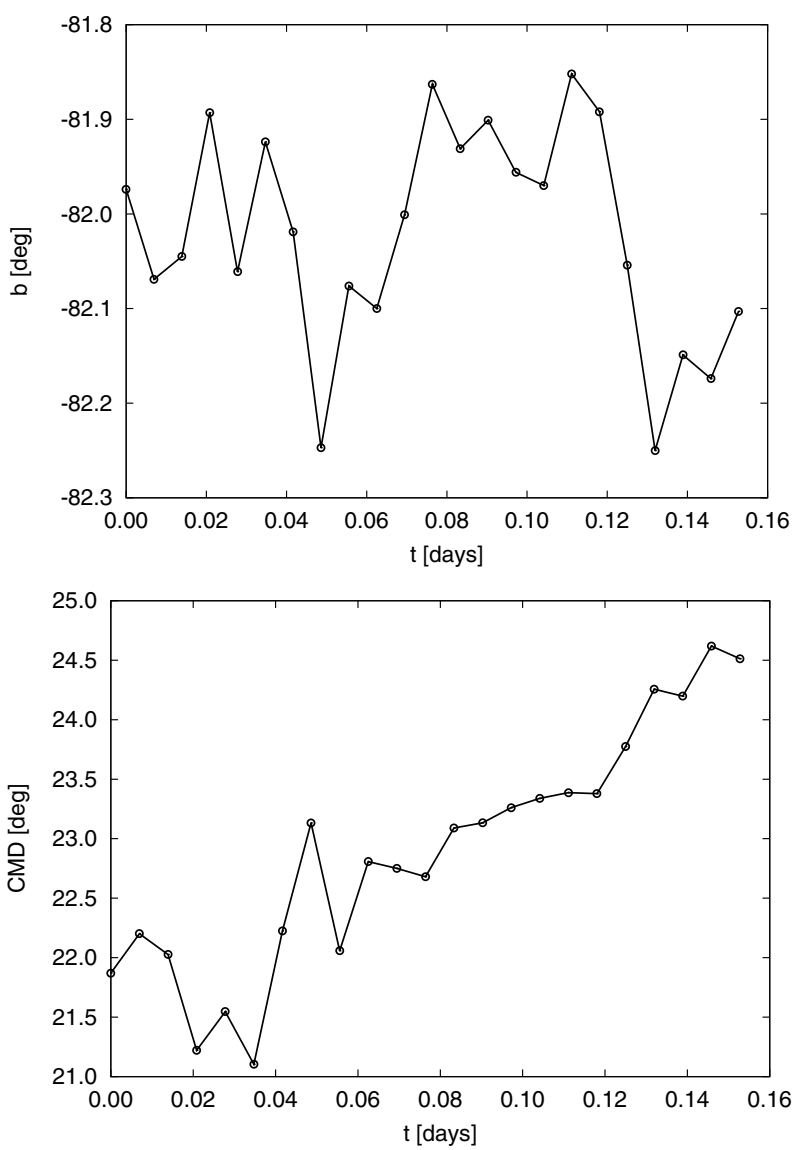

Fig. 2. Motion of a single CBP. Top panel: latitude, $b$, over time, $t$; bottom panel: central meridional distance, CMD over time, $t$.

After completing all the procedures described above, we obtained 906 velocity measurements by tracing CBPs over just two days. Olemskoy \& Kitchatinov (2005) pointed out that nonuniform distribution of tracers can result in false flows. This effect is most notable for meridional motion and rotation velocity residuals, but can easily be removed by assigning the calculated velocity to the latitude of the first measurement of position (Olemskoy \& Kitchatinov 2005; Sudar et al. 2014). Although the effect is negligible for solar rotation, we nevertheless applied the correction in this work.

It is important to keep in mind that even when the tracers are uniformly distributed over the solar surface, the distribution of tracers in latitude will be non-uniform. As we move from the equator to the pole, the area of each latitude bin becomes smaller, so we observe progressively fewer tracers $(\sim \cos b)$.

\section{Results}

In this work, we present an analysis of the motion of CBPs observed by the SDO/AIA instrument. To better understand the results and the potential of future studies along these lines, it is very useful to analyse the accuracy and errors of the dataset.

In Fig. 3 we show the errors of the calculated rotational velocities, $\sigma\left(\omega_{\text {rot }}\right)$, for each CBP, which resulted from errors in the linear fitting of longitude vs time measurements. Although the errors can go up to $3^{\circ} \mathrm{d}^{-1}$, the most are below $1^{\circ} \mathrm{d}^{-1}$. In Fig. 4 we show these errors in heliographic coordinates to check their spatial distribution on the solar surface. Larger errors are shown with brighter shades and we can see that these roughly correspond to the positions of active regions shown in the bottom

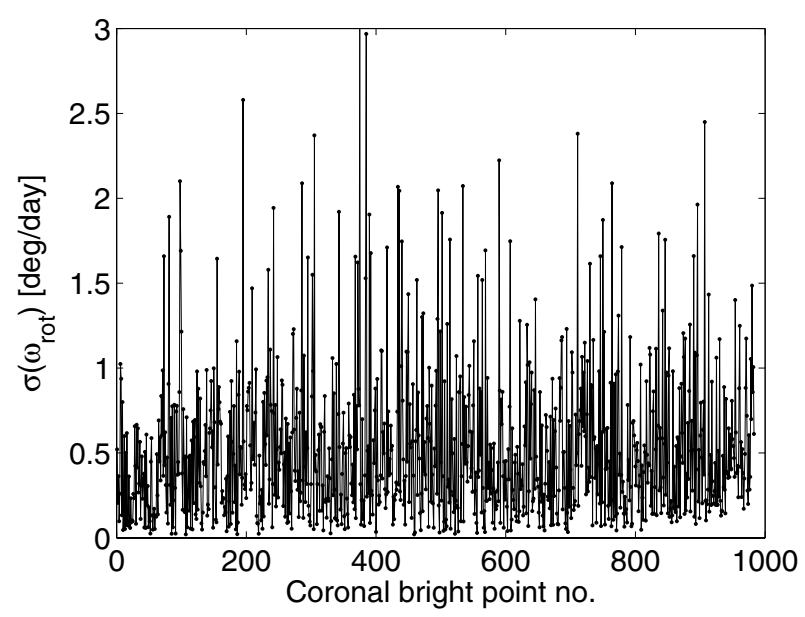

Fig.3. Errors of rotational velocity, $\sigma\left(\omega_{\text {rot }}\right)$, for each of the 906 measurements.

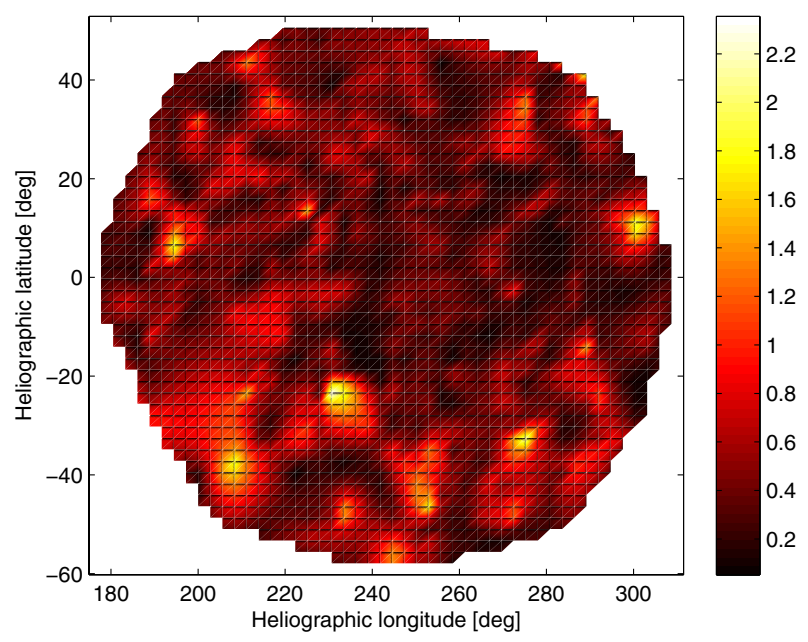

Fig. 4. Distribution of rotation velocity errors $\left(\omega_{\text {rot }}\right)$ in heliographic coordinates. Error scale is in ${ }^{\circ} \mathrm{d}^{-1}$.

panel of Fig. 1. This correlation with active regions is probably a consequence of the design of the detection algorithm and the difficulties in detection of CBPs over a bright, variable background.

In Fig. 5 we show the distribution of CBPs in heliographic coordinates with arrows indicating the velocity vector. As expected, the dominant effect is that of solar rotation. The latitudinal dependence of rotational velocity is usually expressed as (Howard \& Harvey 1970; Schröter 1985):

$\omega_{\text {rot }}(b)=A+B \sin ^{2} b+C \sin ^{4} b$,

where $b$ is the latitude. Parameter $A$ represents equatorial velocity, while $B$ and $C$ depict the deviation from rigid body rotation. The problem with Eq. (3) is that the functions in this expression are not orthogonal, so the parameters are not independent of each other (Duvall \& Svalgaard 1978; Snodgrass 1984; Snodgrass \& Howard 1985; Snodgrass \& Ulrich 1990). This crosstalk among the coefficients is particularity bad for $B$ and $C$. The effect of crosstalk does not affect the actual shape of the fit $\left(\omega_{\text {rot }}(b)\right)$, but it creates confusion when directly comparing coefficients from different authors or obtained by different indicators.

There are various ways to alleviate this problem. Frequently $C$ is set to zero since its effect is noticeable only at higher latitudes. This is almost a standard practice 


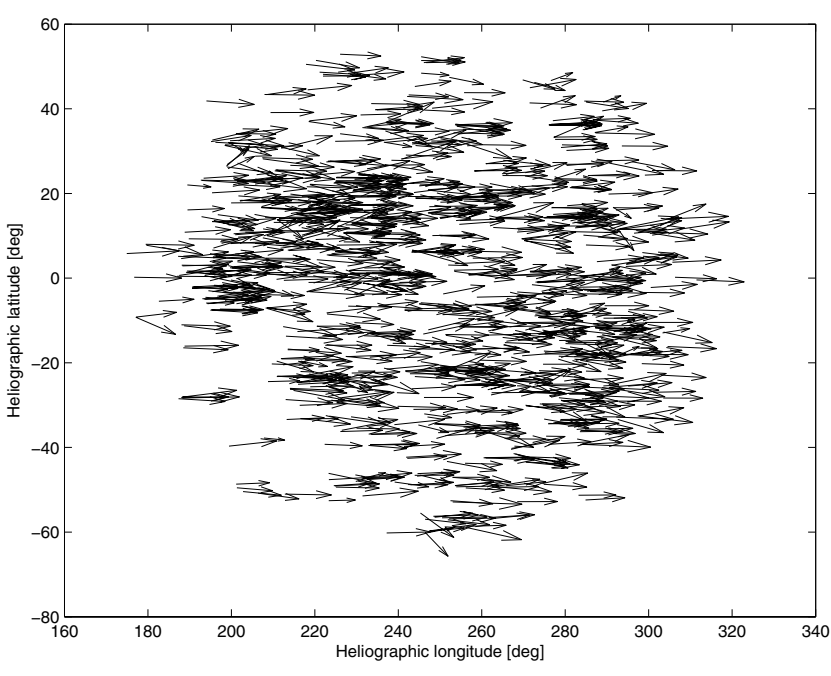

Fig. 5. Distribution of CBPs in heliographic coordinates with arrows showing the direction and strength of the velocity vector.

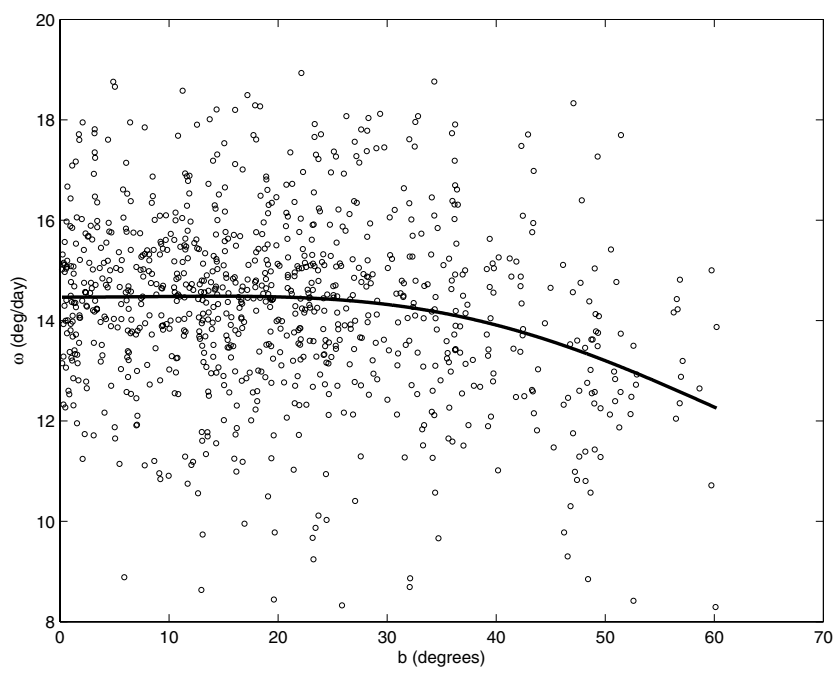

Fig. 6. Solar differential rotation profile obtained with data from SDO/AIA. Open circles are individual measurements, while the solid line is the best fit defined by Eq. (3) for the $A \neq B \neq C$ case.

when observing rotation by tracing sunspots or sunspot groups because their positions do not extend to high latitudes (Howard et al. 1984; Balthasar et al. 1986b; Pulkkinen \& Tuominen 1998; Brajša et al. 2002a; Sudar et al. 2014).

Another way to reduce the crosstalk problem is to set the $C / B$ ratio to some fixed value. Scherrer et al. (1980) set the ratio $C / B=1$ while Ulrich et al. (1988), after measuring the covariance of $B$ and $C$, set the ratio to $C / B=1.0216295$.

In Fig. 6 we show individual measurements of rotational velocities, $\omega_{\text {rot }}$, with respect to latitude, $b$, as open circles. We indicate, with a solid line, the best fit to the data using a functional form given in Eq. (3). Coefficients of the fit are given in Table 1. We also fitted the rotation profile for northern and southern solar hemisphere separately because of possible asymmetry (see e.g. Wöhl et al. 2010) and show the results in the same table. Coefficient $A$ shows a larger value in the Southern hemisphere for all three fit functions. Jurdana-Šepić et al. (2011) reported that coefficient $A$ is larger when solar activity is smaller. The SIDC data (SILSO World Data Center 2011) shows that the northern hemisphere is more active both when looking at
Table 1. Coefficients of the solar rotation profile.

\begin{tabular}{clllc}
\hline \hline Type & $A\left[{ }^{\circ} \mathrm{d}^{-1}\right]$ & $B\left[{ }^{\circ} \mathrm{d}^{-1}\right]$ & $C\left[{ }^{\circ} \mathrm{d}^{-1}\right]$ & $n$ \\
\hline$A, B, C$ & $14.47 \pm 0.10$ & $+0.6 \pm 1.0$ & $-4.7 \pm 1.7$ & 906 \\
$A, B=C$ & $14.59 \pm 0.07$ & $-1.35 \pm 0.21$ & $-1.35 \pm 0.21$ & 906 \\
$A, B, C=0$ & $14.62 \pm 0.08$ & $-2.02 \pm 0.33$ & 0 & 906 \\
\hline \multicolumn{5}{c}{ Northern hemisphere } \\
\hline$A, B, C$ & $14.43 \pm 0.13$ & $+0.8 \pm 1.5$ & $-5.6 \pm 3.0$ & 461 \\
$A, B=C$ & $14.55 \pm 0.10$ & $-1.35 \pm 0.35$ & $-1.35 \pm 0.35$ & 461 \\
$A, B, C=0$ & $14.57 \pm 0.10$ & $-1.92 \pm 0.52$ & 0 & 461 \\
\hline \multicolumn{5}{c}{ Southern hemisphere } \\
\hline$A, B, C$ & $14.50 \pm 0.15$ & $+0.7 \pm 1.4$ & $-4.8 \pm 2.3$ & 445 \\
$A, B=C$ & $14.65 \pm 0.11$ & $-1.39 \pm 0.28$ & $-1.39 \pm 0.28$ & 445 \\
$A, B, C=0$ & $14.69 \pm 0.12$ & $-2.14 \pm 0.45$ & 0 & 445 \\
\hline \multicolumn{5}{c}{}
\end{tabular}

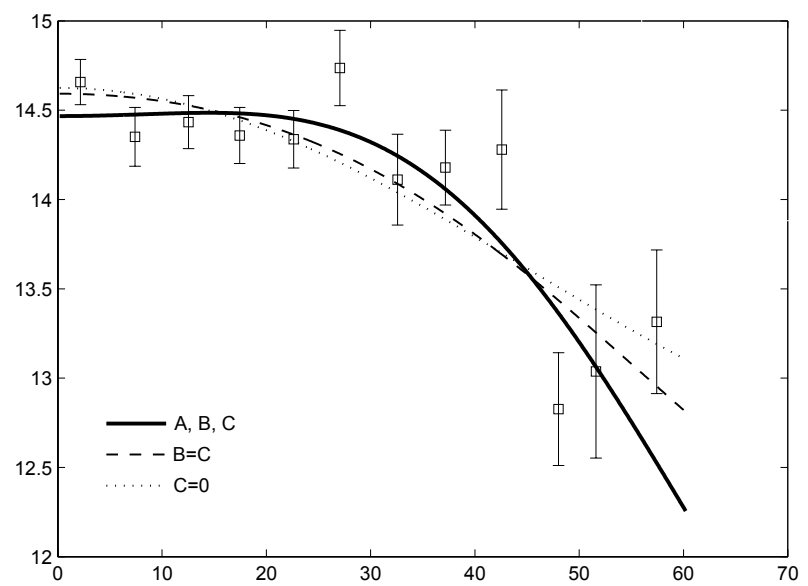

Fig. 7. Comparison of three different fitting procedures for the solar differential rotation profile. Average values of $\omega_{\text {rot }}$ in $5^{\circ}$ bins in latitude, $b$, are also shown with their respective errors.

the monthly smoothed means and the daily sunspot data, which is consistent with Jurdana-Šepić et al. (2011). However, judging by the errors of the coefficients, the difference between south and north is statistically low and the hypothesis that this is a result of asymmetric solar activity needs to be verified with a larger data sample.

In Fig. 7 we show a comparison between different fitting techniques: $A \neq B \neq C$ (solid line), $A \neq B=C$ (dashed line), and $A \neq B, C=0$ (dotted line). In the same figure we also show average values of $\omega_{\text {rot }}$ in bins $5^{\circ}$ wide in latitude, $b$, with their respective errors.

\section{Discussion}

In Table 2 we show a comparison of the solar differential profile (Eq. (3)) from a number of different sources, including the results from this paper (Table 1). Since we found no statistically significant difference between the northern and southern hemisphere we include the results for both hemispheres combined. Results in Table 2 come from a wide variety of different techniques, tracers, and instruments.

Snodgrass (1984) suggested that the rotation profile should be expressed in terms of Gegenbauer polynomials since they are orthogonal on the disk. This eliminates the cross-talk problem between coefficients in Eq. (3). Using the expansion in terms of Gegenbauer polynomials, the solar rotation profile becomes:

$\omega_{\text {rot }}(b)=A_{\mathrm{G}} T_{0}^{1}(\sin b)+B_{\mathrm{G}} T_{2}^{1}(\sin b)+C_{\mathrm{G}} T_{4}^{1}(\sin b)$, 
Table 2. Comparison with some other results.

\begin{tabular}{|c|c|c|c|c|c|c|c|c|}
\hline Method/object & Time period & $A\left[{ }^{\circ} \mathrm{d}^{-1}\right]$ & $B\left[{ }^{\circ} \mathrm{d}^{-1}\right]$ & $C\left[{ }^{\circ} \mathrm{d}^{-1}\right]$ & $A_{\mathrm{G}}\left[{ }^{\circ} \mathrm{d}^{-1}\right]$ & $B_{\mathrm{G}}\left[{ }^{\circ} \mathrm{d}^{-1}\right]$ & $C_{\mathrm{G}}\left[{ }^{\circ} \mathrm{d}^{-1}\right]$ & Ref. \\
\hline CBPs & 1967 & $14.65 \pm 0.2$ & & & 14.65 & & & (1) \\
\hline CBPs & 1994-1997 & $14.39 \pm 0.01$ & $-1.91 \pm 0.10$ & $-2.45 \pm 0.17$ & 13.80 & -0.709 & -0.117 & (2) \\
\hline CBPs & 1998-1999 & $14.454 \pm 0.027$ & $-2.22 \pm 0.07$ & $-2.22 \pm 0.07$ & 13.82 & -0.740 & -0.106 & (3) \\
\hline CBPs & 1998-2006 & $14.499 \pm 0.006$ & $-2.54 \pm 0.06$ & $-0.77 \pm 0.09$ & 13.93 & -0.611 & -0.037 & (4) \\
\hline CBPs & 2011 Jan. 1-2 & $14.47 \pm 0.10$ & $+0.6 \pm 1.0$ & $-4.7 \pm 1.7$ & 14.19 & -0.507 & -0.224 & (5) \\
\hline CBPs & 2011 Jan. 1-2 & $14.59 \pm 0.07$ & $-1.35 \pm 0.21$ & $-1.35 \pm 0.21$ & 14.20 & -0.450 & -0.064 & (5) \\
\hline CBPs & 2011 Jan. 1-2 & $14.62 \pm 0.08$ & $-2.02 \pm 0.33$ & & 14.22 & -0.404 & & (5) \\
\hline sunspot groups & $1853-1996$ & $14.531 \pm 0.003$ & $-2.75 \pm 0.05$ & & 13.98 & -0.550 & & (6) \\
\hline sunspot groups & 1874-1976 & $14.551 \pm 0.006$ & $-2.87 \pm 0.06$ & & 13.98 & -0.574 & & (7) \\
\hline sunspot groups & 1878-2011 & $14.499 \pm 0.005$ & $-2.64 \pm 0.05$ & & 13.97 & -0.528 & & (8) \\
\hline sunspot groups & 1880-1976 & $14.37 \pm 0.01$ & $-2.59 \pm 0.16$ & & 13.85 & -0.518 & & (9) \\
\hline sunspots & 1921-1982 & $14.522 \pm 0.004$ & $-2.84 \pm 0.04$ & & 13.95 & -0.568 & & (10) \\
\hline sunspot groups & 1921-1982 & $14.393 \pm 0.010$ & $-2.95 \pm 0.09$ & & 13.80 & -0.590 & & (10) \\
\hline $\mathrm{H} \alpha$ filaments & 1972-1987 & $14.45 \pm 0.15$ & $-0.11 \pm 0.90$ & $-3.69 \pm 0.90$ & 14.11 & -0.514 & -0.176 & (11) \\
\hline magnetic features & $1967-1980$ & $14.307 \pm 0.005$ & $-1.98 \pm 0.06$ & $-2.15 \pm 0.11$ & 13.73 & -0.683 & -0.102 & (12) \\
\hline magnetic features & 1975-1991 & $14.42 \pm 0.02$ & $-2.00 \pm 0.13$ & $-2.09 \pm 0.15$ & 13.84 & -0.679 & -0.100 & (13) \\
\hline Doppler & 1966-1968 & 13.76 & -1.74 & -2.19 & 13.22 & -0.640 & -0.104 & (14) \\
\hline Doppler & 1967-1984 & 14.05 & -1.49 & -2.61 & 13.53 & -0.646 & -0.124 & (15) \\
\hline Helioseismology & 1996 & 14.16 & -1.63 & -2.52 & 13.62 & -0.662 & -0.120 & (16) \\
\hline Helioseismology & 2002 Apr. & 14.04 & -1.70 & -2.49 & 13.49 & -0.672 & -0.119 & (17) \\
\hline
\end{tabular}

References. (1) Dupree \& Henze (1972); (2) Hara (2009); (3) Brajša et al. (2004); (4) Wöhl et al. (2010); (5) this paper; (6) Pulkkinen \& Tuominen (1998); (7) Balthasar et al. (1986b); (8) Sudar et al. (2014); (9) Brajša et al. (2002a); (10) Howard et al. (1984); (11) Brajša et al. (1991); (12) Snodgrass (1983); (13) Komm et al. (1993); (14) Howard \& Harvey (1970); (15) Snodgrass (1984); (16) Schou et al. (1998); (17) Komm et al. (2004).

where $A_{\mathrm{G}}, B_{\mathrm{G}}$, and $C_{\mathrm{G}}$ are coefficients of expansion and $T_{0}^{1}(\sin b), T_{2}^{1}(\sin b)$, and $T_{4}^{1}(\sin b)$ are Gegenbauer polynomials as defined by Snodgrass \& Howard (1985) in their Eq. (2).

As Snodgrass \& Howard (1985) and Snodgrass \& Ulrich (1990) pointed out, the relationship between coefficients $A, B$, and $C$ from the standard rotation profile (Eq. (3)) and coefficients $A_{\mathrm{G}}, B_{\mathrm{G}}$, and $C_{\mathrm{G}}$ from Eq. (4) is linear. Therefore, it is not necessary to recalculate the fits using Gegenbauer polynomials, we can compute $A_{\mathrm{G}}, B_{\mathrm{G}}$, and $C_{\mathrm{G}}$ directly from $A, B$, and $C$. We used the relationship given in Snodgrass \& Howard (1985; their Eq. (4)) since there seems to be a typo for a similar relationship for coefficients $C$ and $C_{\mathrm{G}}$ in Snodgrass \& Ulrich (1990). In Table 2 we also show the values of coefficients $A_{\mathrm{G}}, B_{\mathrm{G}}$, and $C_{\mathrm{G}}$.

Our rotational profile results are roughly consistent with all the previously published work we surveyed (Table 2). The accuracy of our coefficients is lower when compared with other results, a consequence of our fairly small number of data points ( $n=906)$. Wöhl et al. (2010), for example, had more than 50000 data points spanning a time interval of 8 years. We have used data spanning only 2 days. It is therefore reasonable to expect that with AIA/SDO CBP data we could reach 50000 data points with only 4 months of data and achieve similar accuracy in solar rotation profile coefficients. This means that with AIA/SDO data it should be possible to measure the rotation profile several times per year and track possible changes in solar surface differential rotation directly with a very simple tracer method. This is also true for meridional motion and Reynolds stress, both of which probably vary over the solar cycle (see e.g. Sudar et al. 2014).

\section{Summary and conclusion}

Using $19.3 \mathrm{~nm}$ data from the SDO/AIA instrument at $10 \mathrm{~min}$ cadence we have identified a large number of CBPs, resulting in 906 rotation velocity measurements. We obtained a fairly good differential solar rotation profile even though we used data spanning only two days. The large density of data points in time is a result of several factors. The instrument itself (SDO/AIA) has better spatial resolution and is capable of high cadence $(<5 \mathrm{~min})$. For comparison, SOHO/EIT $28.4 \mathrm{~nm}$ channel had a cadence of two images every $6 \mathrm{~h}$ (Wöhl et al. 2010). In this work, the high cadence enabled us to track and measure velocities of short lived CBPs which could not be detected or accurately tracked by the comparatively large time interval between successive images in the SOHO/EIT $28.4 \mathrm{~nm}$ channel. Coupled with the fact that short lived CBPs are more numerous than long-lived ones (Brajša et al. 2008), this resulted in a very high density of data points in time. High data density necessitated the use of an automatic procedure to detect and track CBPs. The segmentation algorithm used here proved to be completely adequate for the task, as similar algorithms have elsewhere (McIntosh \& Gurman 2005; McIntosh et al. 2014a,b).

The surface rotation profile and its accuracy obtained by helioseismology is seldom given in a form suitable for comparison with those obtained by tracer measurements. We can estimate from the number of published significant digits in the results of Schou et al. (1998) and Komm et al. (2004), given in Table 2, that the accuracy is of the same order as better quality tracer measurements. Zaatri et al. (2009) published the error for the coefficent $B$ (see Eq. (3)) and the value of 0.01 from that paper is in agreement with our estimate above.

It is quite conceivable that errors in the differential rotation profile coefficients will drop significantly when more data is used. From our analysis, we can expect to obtain 400-500 velocity measurements per day from CBPs using SDO/AIA. A time interval of 4 months seems adequate to obtain 50000 velocity measurements, which should be sufficient to match the most accurate results obtained by tracer methods (for example Wöhl et al. 2010).

Coronal bright points are also very good tracers since they extend to much higher latitudes than sunspots. They are also quite numerous in all phases of the solar cycle while sunspots are often absent in the minimum of the cycle. 
This opens up an intriguing possibility of measuring the solar rotation profile almost from one month to the next over an entire cycle. Such studies could provide new insight into the mechanisms responsible for solar rotation. We already know that meridional motion exhibits some changes during the course of the solar cycle, and the same is probably true for Reynolds stress. Sudar et al. (2014) found by averaging almost 150 years of sunspot data that meridional motion changes slightly over the solar cycle and hinted that the Reynolds stresses are probably changing too. Here we have found a small asymmetry in rotation profile for two solar hemispheres and suggested that this might be related to different solar activity levels in the two hemispheres. This needs to be verified with a larger dataset, however, as the difference in rotation profiles was of low statistical significance.

The planned SDO mission duration of 5-10 years will cover a large portion of the solar cycle which should result in an enormous amount of velocity data to assist in the understanding of the nature and variation of solar rotation profile. Having more detailed temporal resolution and direct results (without the need to average many solar cycles) could prove to be very informative.

A time interval of 10 min between successive images also offers a good opportunity to study the evolution of CBPs and the possible effect this might have on the detected surface velocity fields. For example, Vršnak et al. (2003) reported that longer lasting CBPs show different results than short-lived CBPs.

Based on the promising results here, we will use larger datasets to further exploit the potential of SDO/AIA CBP data to determine meridional motions, rotation velocity residuals, Reynolds stresses, and proper motions in subsequent papers.

Acknowledgements. This work has received funding from the European Commission FP7 project eHEROES $(284461,2012-2015)$ and SOLARNET project $(312495,2013-2017)$ which is an Integrated Infrastructure Initiative (I3) supported by FP7 Capacities Programme. It was also supported by the Croatian Science Foundation under the project 6212 Solar and Stellar Variability. SS was supported by NASA Grant NNX09AB03G to the Smithsonian Astrophysical Observatory and contract SP02H1701R from Lockheed-Martin to SAO. We would like to thank the SDO/AIA science teams for providing the observations. We would also like to thank Veronique Delouille and Alexander Engell for valuable help in the preparation of this work.

\section{References}

Balthasar, H., Lustig, G., Woehl, H., \& Stark, D. 1986a, A\&A, 160, 277 Balthasar, H., Vázquez, M., \& Wöhl, H. 1986b, A\&A, 155, 87 Beck, J. G. 2000, Sol. Phys., 191, 47

Brajša, R., Vršnak, B., Ruždjak, V., Schroll, A., \& Pohjolainen, S. 1991, Sol. Phys., 133, 195

Brajša, R., Wöhl, H., Vršnak, B., et al. 2001, A\&A, 374, 309

Brajša, R., Wöhl, H., Vršnak, B., et al. 2002a, Sol. Phys., 206, 229

Brajša, R., Wöhl, H., Vršnak, B., et al. 2002b, A\&A, 392, 329
Brajša, R., Wöhl, H., Vršnak, B., et al. 2004, A\&A, 414, 707

Brajša, R., Wöhl, H., Vršnak, B., et al. 2008, Central Europ. Astrophys. Bull., 32,165

Dorotovič, I., Shahamatnia, E., Lorenc, M., et al. 2014, Sun and Geosphere, 9,81

Dupree, A. K., \& Henze, Jr., W. 1972, Sol. Phys., 27, 271

Duvall, Jr., T. L., \& Svalgaard, L. 1978, Sol. Phys., 56, 463

Erwin, E. H., Coffey, H. E., Denig, W. F., et al. 2013, Sol. Phys., 288, 157

Hara, H. 2009, ApJ, 697, 980

Howard, R. 1984, ARA\&A, 22, 131

Howard, R., \& Harvey, J. 1970, Sol. Phys., 12, 23

Howard, R., Gilman, P. I., \& Gilman, P. A. 1984, ApJ, 283, 373

Howe, R. 2009, Liv. Rev. Sol. Phys., 6, 1

Jurdana-Šepić, R., Brajša, R., Wöhl, H., et al. 2011, A\&A, 534, A17

Karachik, N., Pevtsov, A. A., \& Sattarov, I. 2006, ApJ, 642, 562

Kariyappa, R. 2008, A\&A, 488, 297

Komm, R. W., Howard, R. F., \& Harvey, J. W. 1993, Sol. Phys., 145, 1

Komm, R., Corbard, T., Durney, B. R., et al. 2004, ApJ, 605, 554

Kosovichev, A. G., Schou, J., Scherrer, P. H., et al. 1997, Sol. Phys., 170, 43

Lemen, J. R., Title, A. M., Akin, D. J., et al. 2012, Sol. Phys., 275, 17

Lorenc, M., Rybanský, M., \& Dorotovič, I. 2012, Sol. Phys., 281, 611

Martens, P. C. H., Attrill, G. D. R., Davey, A. R., et al. 2012, Sol. Phys., 275, 79

McIntosh, S. W., \& Gurman, J. B. 2005, Sol. Phys., 228, 285

McIntosh, S. W., Wang, X., Leamon, R. J., et al. 2014a, ApJ, 792, 12

McIntosh, S. W., Wang, X., Leamon, R. J., \& Scherrer, P. H. 2014b, ApJ, 784, L32

Newton, H. W., \& Nunn, M. L. 1951, MNRAS, 111, 413

Olemskoy, S. V., \& Kitchatinov, L. L. 2005, Astron. Lett., 31, 706

Ossendrijver, M. 2003, A\&ARv, 11, 287

Pulkkinen, P., \& Tuominen, I. 1998, A\&A, 332, 755

Roša, D., Vršnak, B., \& Božić, H. 1995, Hvar Observatory Bulletin, 19, 23

Roša, D., Vršnak, B., Božić, H., et al. 1998, Sol. Phys., 179, 237

Rozelot, J.-P., \& Neiner, C. 2009, The Rotation of Sun and Stars (Berlin: Springer Verlag), Lect. Notes Phys., 765

Rüdiger, G., \& Hollerbach, R. 2004, The Magnetic Universe (Wiley Interscience)

Scherrer, P. H., Wilcox, J. M., \& Svalgaard, L. 1980, ApJ, 241, 811

Schou, J., Antia, H. M., Basu, S., et al. 1998, ApJ, 505, 390

Schröter, E. H. 1985, Sol. Phys., 100, 141

SILSO World Data Center 2011, International Sunspot Number Monthly Bulletin and online catalogue

Skokić, I., Brajša, R., Roša, D., Hržina, D., \& Wöhl, H. 2014, Sol. Phys., 289, 1471

Snodgrass, H. B. 1983, ApJ, 270, 288

Snodgrass, H. B. 1984, Sol. Phys., 94, 13

Snodgrass, H. B., \& Howard, R. 1985, Sol. Phys., 95, 221

Snodgrass, H. B., \& Ulrich, R. K. 1990, ApJ, 351, 309

Stark, D., \& Wöhl, H. 1981, A\&A, 93, 241

Stix, M. 2004, The Sun: an introduction, 2nd edn., Astron. Astrophys. Libr. (Berlin: Springer)

Sudar, D., Skokić, I., Ruždjak, D., Brajša, R., \& Wöhl, H. 2014, MNRAS, 439, 2377

Ulrich, R. K., Boyden, J. E., Webster, L., Padilla, S. P., \& Snodgrass, H. B. 1988, Sol. Phys., 117, 291

Vršnak, B., Brajša, R., Wöhl, H., et al. 2003, A\&A, 404, 1117

Wilcox, J. M., \& Howard, R. 1970, Sol. Phys., 13, 251

Willis, D. M., Coffey, H. E., Henwood, R., et al. 2013a, Sol. Phys., 288, 117

Willis, D. M., Henwood, R., Wild, M. N., et al. 2013b, Sol. Phys., 288, 141

Wöhl, H., Brajša, R., Hanslmeier, A., \& Gissot, S. F. 2010, A\&A, 520, A29

Zaatri, A., Wöhl, H., Roth, M., Corbard, T., \& Brajša, R. 2009, A\&A, 504, 589 